\title{
The interplay between global standards and local practice in nursing
}

Torbjørg Meum ${ }^{\mathrm{a}}$, Gunnar Ellingsen ${ }^{\mathrm{a}}$, Eric Monteiro ${ }^{\mathrm{b}}$ Gro Wangensteen $^{\mathrm{c}}$, Harald Igesund ${ }^{\mathrm{c}}$

${ }^{a}$ Telemedicine Research Group, Department of Clinical Medicine, University of Tromsø.

${ }^{b}$ Department of Computer and Information Systems, Norwegian University of Science and Technology, Trondheim, Norway

${ }^{c}$ University Hospital of North Norway

Abstract: 
Purpose: The paper assesses the extent, form, and transformation of global nursing classifications (NANDA) in a nursing practice during a period of five years.

Method: Mixed-method: a) quantitative frequency analysis and b) qualitative (longitudinal case study)

Results: A surprisingly high proportion (95.5\%) of nursing diagnoses were consistent with the global standard, in spite of the users' relative freedom in the practice of coding. This is elaborated more thoroughly through a co-constructing perspective, emphasizing how the global standard and the practice mutually shaped each other over several years.

Conclusion: Standardization is an iterative process that is performed in close relationship with practice. The mutual interrelation between formal classifications (NANDA) and local practices are co-constructed in a dynamic interplay that evolves over time. In such a process, the use of local classifications may be a means to bridge the gap between global standards and local distinctiveness.

Keywords: Nursing classifications, Standardization, Electronic Patient Records.

What was known before the study:

Comparing and cross mapping between different nursing terminologies shows that none of the existing terminologies cover all the needs of local nursing practice.

Alignment of standards to the existing socio-technical infrastructure is needed as a means for bridging the gap between interoperability and domain-specific multiplicity

What the study has added to the body of knowledge:

Empirical insight on how standardization and work practice is co-constructed in a dynamic interplay over several years.

Elaboration on how the use of local classifications (folksonomies) bridged the gap between global standards and local practice.

A careful use of local classifications may serve as a facilitator mechanism when implementing global classifications in organizations. 


\section{Introduction}

"First, do no harm," derived from the Hippocratic Oath, is one of the fundamental principles for health care around the world. However, medical errors and injuries have caused growing concern in every society in modern times. According to the Institute of Medicine, a significant number of hospitalized patients in the United States are exposed to medical errors where miscommunication or lack of communication was a leading cause of adverse errors, i.e. injury caused by medical management rather than the underlying condition of the patient $[1,2]$. Similarly, a recent survey by the Ministry of Health and Care Service in Norway showed that approximately $14,5 \%$ of all hospitalizations were associated with patient injury ${ }^{1}$. Internationally and nationally there has been a lot of "promise" associated with how ICT can be used for enhancing the quality and safety in health care sector. Particularly, computer systems are expected to streamline care processes through the use of decision support and performance measurements [1]. However, although some studies have shown promising results for standalone systems such as bar-code technology [3], other studies have revealed unintended consequences [4], workarounds [5] and generation of new errors [6]. Some of the difficulty is ascribed the complexity of the health care environment. The transition from paper-based to computer-based support of the clinical workflow is not a straightforward matter, as it involves collaboration between different professionals, is distributed across time and space, and is an interplay between multiple tasks and supportive artifacts [7].

Standardisation in a wide sense has been considered a key means to deal with these challenges [8-10]. Standardised ICT, protocols, terminologies and practices are all considered to be vehicles for improved quality and patient safety [1]. Health care processes heavily depend on both information and knowledge and better management of health information is a prerequisite to achieving patient safety as a standard of care [9, p. 4]. Following the evidence-based practice movement, the ability to formalization, rationalization and streamline work practices using "universal" standards has been emphasized as a way to replace inefficiency and variation with quality and standardisation

${ }^{1}$ Unpublished report, available on: http://www.regjeringen.no/nb/dep/hod/aktuelt/nyheter/2011/midlertidig.html?id=659472 
$[11,12]$. A number of studies have stressed the role and utility of performance standards incorporated in computerized decision-support systems such as clinical guidelines, clinical pathways, computerized physician order entry (CPOE), care plans, reminders and alerts [5, $7,13]$. Moreover, dimension of information quality in information systems is typically measured by completeness and accuracy [10]. Consequently, standardised terminologies and semantic interoperability are considered important prerequisites for integrated EPR systems to enhance the quality of the care process [9].

In health care, organizations such as the International Standardization Organisation (ISO) prepare formal standards, guidelines and recommendation. ISO has traditionally designed technical standards, but in recent years, it has prepared functional standards for the electronic patient record (EPR) [14] as well as an integrative model for nursing terminologies [15]. In addition, professional communities in nursing like the International Council of Nursing $(\mathrm{ICN})^{2}$ and North American Nursing Association (NANDA) ${ }^{3}$ has played a key role in developing purpose-specific terminologies for nursing practice.

However, like other standardization efforts in healthcare [16-18], adopting and using standardized terminologies in nursing practice has been a cumbersome and slow process [19], and notoriously difficult to realize [20]. Although some studies have shown correlation between the use of nursing terminologies and quality of documentation [21], other studies have demonstrated that the diversity of terminologies used in nursing as an obstacle of semantic interoperability [20, 22-24]. Moreover, a European survey revealed that general use of nursing terminologies is still lacking which makes access to nursing data an obstacle [19].

Still we are worried about a simplistic debunking the implementation of nursing terminologies as "success" or "failures" based on snap-shot case studies or surveys. Accordingly we base our study on an extended methodological where we combine a 5-year longitudinal interpretive case study with a quantitative frequency analysis. Firstly, we

${ }^{2}$ http://www.icn.ch/

${ }^{3}$ http://www.nanda.org/ 
illustrate how sustainable use of nursing terminologies is a co-construction between the global terms and local practice and how this presupposes a long-term negotiation between these two. Secondly, based on a frequency analysis, we present an interesting result where as much as $95,5 \%$ of all nursing diagnoses was consistent with NANDA. We analyze how this underpin our argument on co-construction, and also provide a critical reflection on the limitation of this method. Thirdly, we discuss the need for some leeway: Some degree of "slack" is necessary for any standard to maintain local innovation and domain specific knowledge. We argue that such kind of "slack" facilitate consistency and accuracy of the documentation in the EPR and suggest the use of social tagging (folksonomies) in combination with standardized terminologies as a way to resolve the tension between standardization and flexibility in the use of nursing terminologies.

Our empirical materials draws on a longitudinal (2005 - 2010) case study in which we applied a mix method to explore the implementation and use of electronic care plans and nursing classifications in a psychogeriatric ward at the University Hospital in Norway, i.e., a frequency analysis of NANDA, mapping between NANDA and free-text as well as an ethnographically inspired case study. Mapping between different terminologies, or between free text and terminologies, has been a key measure for the assessment of sematic interoperability in nursing documentation [25]. However, in this study we have not just focused on the scope and correlation between NANDA and free-text diagnoses, but also how context-specific nursing diagnoses and interventions evolve in everyday practice.

\section{Background and status on standardization in nursing}

Since the release of the Institute of Medicine (IOM) report "To Err is human: Building a safer Health Care System [1], efforts have been aimed towards developing and adoption of information systems to support and improve patient care. A health information infrastructure is regarded as a foundation for a safer health care and highlights health data interchange, terminologies, and medical knowledge representation key capabilities to promote patient safety and improved quality. The emphasis on standardisation in nursing is, in part, an expression of this broader trend where standards are considered the building blocks to share and compare information among care teams and facilitating the use of decision-support in clinical information systems.

Standardization in health care is a powerful movement as "standards specify how we work, how our technology interacts; they hold our socio-technical societies together" [26, p. 8]. Standardization is rooted in strategies as a means to increase the quality and effectiveness 
in health care. The International Organization for Standardization (ISO) Technical report highlights the ability to share information as one of the greatest potential benefits of the EPR and emphasizes interoperability across systems as a major challenges [14].

Timmermans and Berg [26] distinguishes between four standards used in health care; design standards, performance standards, terminological standards, and procedure standards. Design standards are more or less specification of social and technical systems ensuring uniformity and compatibility. Performance standards represent outcome specification used to regulate professional work and outcome measurements. An example is the adoption of quality indicators in Norway that offers transparency that is essential for securing accountability for health systems performance. The third kind of standard are terminologies that have a long history in various levels of the health care, such as professional organizations and the World Health Organization. North American Nursing Diagnosis Association (NANDA) and International Classification of Diseases (ICD) are examples of such standards that have evolved over a long time in order to share and compare clinical information and to ensure stability of meaning over different sites and times. Finally, procedural standards are specifications of processes such as clinical guidelines, protocols, procedures or care plans. These standards demarcate a number of actions to be performed when specified situation and conditions occurs, for example, what step a nurse should follow in preventing decubitus ulcers. All of the above standards may overlap each other and be a part of the same standard. For example, terminologies can be embedded in care plans, and clinical guidelines may also include outcome specifications.

The development of standardized terminologies for nursing started in the 1970s. The North American Nursing Diagnosis Association (NANDA) has been a driving force in efforts to develop diagnostic classification in nursing as a means to support clinical judgement and knowledge development [27]. Similarly, Nursing Intervention Classification (NIC) have been developed and used along with NANDA to support documentation of the phases of the nursing process. The implementation of EPRs including nursing care plans in the recent years has made wide-spread use of standardized nursing terminologies possible.

Moreover, evidence-based approaches in health care focus on uniformity and quality control by streamlining processes [26]. This movement is both a cause and effect of standardized terminologies. Shareable and comparable nursing data is a first step to identify and implement "best nursing practices" [28]. For example, the concepts of NANDA are grounded in evidence-based practices and are updated every second year through the submission of new concepts that are reviewed, verified, and presented at the NANDA conference and published by the NANDA International organization [29]. 
Standardization and integration are key issues to interconnect different layers of the EPR system [30] and facilitate coordination of work practices within and across the care team [31]. This implies the need to maintain the domain-specific knowledge embedded in nursing and, at the same time, enable integration with local practices and applications present in the EPR system.

Despite efforts to develop standardised terminologies, much of nursing's documentation is still captured in narrative text [32]. A recent European survey showed that general use of terminologies is still lacking, which makes access to nursing data and quality of care an obstacle [19]. Some of the reasons for slow and patchy standardisation in nursing are related to professional and political issues as well as the contextual nature of clinical information [26, 33]. Some nursing professionals remain deeply sceptical of the ambition of standardisation, as they see it particularly harmful to the traditional, holistic orientation of nursing [33] and the inherent simplifications and abstractions are seen as undermining the very identity of being a nurse. Other scholars have also emphasized aspects of nursing that do not fit in formal classification systems[34] and how the cultural (American) bias of NANDA makes it unfitting in other countries and cultures [35]. Along these lines, many studies have compared different systems and identified criteria to assess and validate terminologies [21, 24, 36]. Similar studies illustrate that not all criteria are met [22, 37-39] and support the earlier study conducted by Bakken et al. [22], which found that none of the existing systems met all the criteria for complete, comprehensive, clear, and non-redundant representations of concepts. We acknowledge these findings, but rather than see this as a shortcoming of a terminology system, we see this as more or less a general characteristic as it is not possible to develop a system that fits all aspects of nursing care. Instead we need to consider strategies to overcome the misfits and the gaps, implying that some flexibility in use of a global standard is imperative. This takes to a co-construction perspective.

Previous studies on the design and use of nursing terminologies have mainly considered terminologies as static, isolated features in its own right. However given the slow diffusion of terminologies, attention has, in recent years, been directed to the central role of the human, social, and organizational context in which information systems operate. Scholars have focused on the standardisation process as a dynamic interrelationship and negotiation between technology and its social environment, the interplay between universal standards and local practices, and the transformative power of standards in a socio-technical network, i.e. co-construction $[26,30,34]$. The development of a standard in a local practice is therefore one of co-construction in which standardisation and work practice mutually shape and constitute each other [40]. 
Carlile (2004) uses the framework of transferring, translating, and transforming to illustrate the complex processes necessary to share and access domain-specific knowledge [41]. The study illustrates how complexity increases as we move from a syntactic to a pragmatic boundary, and the increased effort that is required for sharing and assessing domainspecific knowledge, as circumstances at a boundary grow more complex. From this point of view, a universal standard is not a straightforward issue, but an iterative transformation process that requires the ability and capability to create new agreements and make changes where needed [41].

A key issue of these studies is how standardised terminologies become embedded in social structures in which knowledge is constituted through a variety of social and political processes [42]. Accordingly, standardisation is not just a fixed feature of an object, but an iterative transformation, i.e., co-construction in an interrelated socio-technical network. Several studies have illustrated the co-construction of standards [see for instance, 40] and described this kind of "tinkering" as a prerequisite for the standard to function in practice [43]. There are, however, few studies that have focused on the mutual dependency between terminologies and local co-construction, and how it evolves in practice over many years as our study does.

Still, due to its origin in science and technology studies [26, 34, 40], a perspective on coconstruction has seldom been underpinned by quantitative data. Quantitative data such as frequency analysis may provide insight about the scope of use of terminology in a specific context. However, clinical information is entangled with its context of production [44] and requires additional work such as translating and sorting according to the situational practice. Several studies have pointed out the necessity of alternative ways to classify the diversity of health care information in general and nursing in particular [34]. These scholars highlight the trade-off between pre-defined categories and the cases that do not fit into formal classification systems. Similar studies have emphasized both the enabling and constraining characteristics of classifications [45], as well as the tension between interoperability and local usability [46]. On the one hand, formal terminologies enable shared meaning and comparability across different contexts. On the other hand, their use restricts activity that does not conform to the types recognized in the category systems [45]. An emerging approach for managing the tension between formal terminologies and local diversity is the use of folksonomies, i.e., user generated metadata or tags [46, 47]. The origin of this "grassroots" categorization comes from social web communities, where users started to tag content on websites. It is part of a new generation of tools for the retrieval, deployment, representation, and production of information, commonly termed Web 2.0. 
[48]. In contrast to formal, predefined classification systems that are relatively static, folksonomies are highly flexible and dynamic. Firstly, there is a mutual dependency between the development of collaborative information services (tags), the production of user-generated content, and the increased usage of folksonomies. Secondly, users create their own tags instead of pre-defined classifications, and thereby allow users to implement their own terminology for indexing and representing content [ibib.48 p.3]. Such bottom-up strategies are the compromise between the hierarchical meta-model and folksonomies, and they support the distribution of information to those persons who are actually doing the work [47, p. 444].

\section{Research Method}

We used a mixed method to explore the scope of standardized terminologies in local practice and the correlation between formal terminologies and local use. The quantitative data from the frequency analysis was used as a part of the contextual background for our interpretative approach for assessing the extent of the phenomenon in question. Along with the quantitative data, the interpretive approach enabled exploration of issues involved in adoption and use of nursing terminologies aiming at producing an understanding of the mutual influence between terminologies and its context that occurs over time [49]. An interpretive case study is an appropriate method to explore how people make sense of classification in the context of use as it asserts that reality is socially constructed and hence cannot be understood independently of the social actors who construct and make sense of this reality [50, p. 13]. Moreover, this approach is also useful to study the content, context, and process of organizational change when researching adoption of nursing terminologies [49]. In line with an interpretive approach, the study adheres to the methodological principles outlined by Klein and Meyers (1999), in terms of preparing the research questions, data collection, and analysis [51]. First, selection of the case was motivated by one author's involvement in an ongoing, longitudinal research project based on the implementation and use of nursing documentation in Norway. Second, the empirical setting was selected because two of the authors were involved in the local project and had in-depth knowledge of the context as well as the historical development of nursing documentation. Together with the other researchers, this provided for an insider's knowledge with an outsider's view [52]. Thirdly, the particular ward had used standards since 2005 and thus gave the opportunity to explore how the standards evolved over time. 


\subsection{Research context}

The study was carried out at the psychogeriatric ward at a University Hospital in Norway. This is an inpatient ward with 14 beds, which treats patients who are suffering from psychiatric disorders including depression, psychosis, dementia, and anxiety disorders. The problems facing patients are complex, since most of them suffer from somatic illnesses in addition to psychiatric illnesses. The work at the ward is highly interdisciplinary, and nurses are believed to play a key role in observing and monitoring patients' needs, as well as assessing patients' cognitive abilities for self-care. The clinical staff comprises psychiatrists, physicians, psychologists, nurses, assistant nurses, social workers, occupational therapists, physiotherapists, and some unskilled staff.

Implementation of the electronic nursing module took place in 2005 as an integral part of the EPR and has been in used continuously thereafter. The main motivation for implementation of the nursing module was based on the need to improve the content and language of nursing documentation, which had been on the agenda for a long time. Information sharing in the traditional, paper-based patient record was characterized by a chronological, sequential report of each shift, where the use of narratives, as well as an unstructured language, was prominent. The management of the ward regarded the use of classifications as a means to provide a language for describing both planned and performed care in their everyday work practice. Thus, the motivation to change from paper to electronic documentation was high and strategies for user training and guidance were prioritized during the implementation.

In line with the majority of Norwegian hospitals, standardized terminologies has played a key part in the adoption and use of a nursing module in Norway as an integrated part of EPR. The development of the nursing module was initiated in 2001 as a joint project between the Norwegian nursing community and the largest Norwegian EPR vendor. Due to the increased international and national attention to the use of standards, it was decided to translate international classifications and make these available in the nursing module. Thus, standardized nursing terminologies are widely used in Norwegian hospitals.

\subsection{Data collection and analysis}

Data collection has been carried out as an iterative process involving multiple data sources (qualitative and quantitative), aimed at corroborating the same phenomenon [53] i.e. how classification has been used in the local context. One of the key tasks in interpretive research is seeking meaning in the social and historical contexts [51]. Accordingly, data collection included a combination of archival documents (frequency analysis), participant observation, and semi-structured interviews. Moreover, data collection was performed in 
collaboration with two project nurses and researchers. As part of an internal evaluation of the new system these two nurses extracted anonymous reports from the EPR systems, which provided an overview of frequency usage of NANDA diagnoses and free-text diagnoses This quantitative frequency analysis provided useful information about the distribution and use of standardized nursing terminologies. Although these data are quantitative in nature, they have been used as part of a "whole" and to corroborate other data sources. Specifically, we conducted a comparison between NANDA and local classifications used in the nursing module during a 2-year period. First, a report generator in the EPR system was used to extract the number of NANDA classifications and the number of free text classifications used during a two-year period. Second, we carried out a mapping between NANDA diagnosis and local diagnosis based on the same criteria as outlined by Zielstorff et.al [54] such as "Same", "Similar", "Broader" and "Narrower" .

The qualitative data collection was based on a previous study by the second author, which lasted from 2005 to 2008. As a continuation of this work, the first author carried out 200 hours of observation of the work practices of nurses and social workers in the period between 2008 and 2010. The focus of the observation study was to gain insight into how the electronic care plan was used in practice and how it evolved over time. To gain additional insight, 13 semi-structured interviews and 6 open-ended interviews were carried out, each lasting from half an hour to an hour a half. The focus of the interviews was how nurses and social workers created a care plan, how they searched for and selected nursing diagnoses and interventions, how often they used the care plan during their shifts, and how it supported and coordinated work practices. The attention was on how the users experienced the use of the system and whether it made sense in everyday practice. All of the interviews were taped and transcribed. Along with the frequency reports and the mappings, field notes and the transcribed interviews constituted the basis for analysis where data have been systematized in relation to key elements of the situation of inquiry.

Data collection and analysis have been regarded as an iterative process, moving "from a precursory understanding of the parts to the whole and from the global understanding of the whole process back to an improved understanding of each part " [51, p. 71]. We have aimed to obtain a historical and contextual understanding of the use of standardized terminologies in nursing, and traced the process from training to everyday use in different contexts. The analysis has been carried out in several stages. The first was the analysis of the mappings that was performed by the second and third author. The next step was the analysis of data from interviews and observation that was conducted by the first and second author. Finally, the different data source was then analyzed as part of a whole to 
complement different perspectives on the situation. In addition to interview data from key personnel, we used official reports and documents. Events and milestones were highlighted, and they became the starting point for questions in the interview guide. This part of the analysis was also an iterative process in which transcribed data were highlighted, discussed, leading to further focus in the next interview, and so on. The different data sources (interviews, observations, documents) provided opportunities for systematic comparison of multiple perspectives on events and processes. In addition, it gave the opportunity to identify and uncover tacit assumptions and "invisible" practices that are often taken for granted [52].

Our previous knowledge of standardization as well as our previous experience was the starting point of the analysis. Three of the authors have experience as nurses, and thus have an insider's knowledge with an analytic outsider's view [52]. In interpretive research, prior knowledge and preconceptions are not considered bias, but are the necessary starting point of our understanding [51, p. 76]. Our prior knowledge and experience provided the lenses, and a sensitizing device, through which our field data were constructed. Furthermore, our understanding was expanded and revised through interaction with the participants in our fieldwork, where issues such as enabling and constraining aspects of standardization were discussed and the co-constructive perspective gradually emerged.

Throughout the study, the participating researchers discussed data from the case for the purposes of critical thinking and reflection in the interpretation process [51]. Similarly, the combination of observation, interviews and document analysis provided the opportunity for reflection, elaboration and clarification of narratives collected from the participants.

\section{Analysis of findings}

\subsection{Implementation of the nursing care plan in 2005}

In 2003 the hospital introduced a new EPR system, which also included a nursing module or a nursing care plan. The nursing module has a pivotal position in the EPR, and is a presumption for using international classifications such as NANDA and NIC, which had been translated to Norwegian. The care plan and some NANDA and NIC codes are illustrated in Figure 1. 


\begin{tabular}{|c|c|c|c|c|c|c|c|c|c|c|c|c|}
\hline New diagnosis.. & New interv... & New order... & \multirow[t]{2}{*}{ Revise... } & Discont. & Up & Down & \multirow{2}{*}{ Std. plan... } & \multirow{2}{*}{$\begin{array}{l}\text { Former plan } \\
\text { Start }\end{array}$} & \multirow{2}{*}{$\begin{array}{l}\text { Open doc } \\
\text { End }\end{array}$} & \multicolumn{3}{|c|}{$\Gamma$ View discontir } \\
\hline \multicolumn{3}{|c|}{ Care plan elements } & & \multicolumn{3}{|c|}{\begin{tabular}{l|l|l} 
NC & Frequency/situation \\
\end{tabular}} & & & & Status & & $\Delta$ \\
\hline \multicolumn{13}{|c|}{ - Care plan diaqnoses } \\
\hline \multicolumn{4}{|c|}{ Ineffective Breathing Pattern } & 3 & & & & 20.05 .06 & & Active & & \\
\hline \multicolumn{4}{|c|}{ Risk for Constipation } & 5 & & & & 20.05 .06 & & Active & & \\
\hline \multicolumn{4}{|c|}{ Bathing/Hygiene Self-Care Deficit } & 7 & & & & 20.05 .06 & & Active & & \\
\hline \multicolumn{4}{|c|}{ Risk for Activity Intolerance } & 3 & & & & 20.05 .06 & & Active & & \\
\hline \multicolumn{4}{|c|}{$\begin{array}{l}\text { Deficient knowledge [specify] ..... 0m } \\
\text { sykdommen,komplikasjonsiare, kosthold og livsiørsel } \\
\text { etterpå }\end{array}$} & 2 & & & & 20.05 .06 & & Active & & \\
\hline \multicolumn{13}{|c|}{ Care plan interventions/orders } \\
\hline \multicolumn{4}{|c|}{ Cardiac Care: Rehabilitative } & 3 & & & & 20.05 .06 & & Active & & \\
\hline \multicolumn{4}{|c|}{ OBS how much activity pat. tolerates } & \multicolumn{2}{|c|}{ Continous } & & & 20.05 .06 & & Active & & \\
\hline \multicolumn{4}{|c|}{ Increase activity level gradually, - see list } & \multicolumn{4}{|c|}{ According. to activity list } & 20.05 .06 & & Active & & \\
\hline \multicolumn{4}{|c|}{$\begin{array}{l}\text { Offer pat help to personal hygiene relatede to pat } \\
\text { condition and activity progression }\end{array}$} & \multicolumn{4}{|c|}{ PRN } & 20.05 .06 & & Active & & $\nabla$ \\
\hline \multicolumn{4}{|l|}{1} & & & & & & & \multicolumn{3}{|c|}{ - } \\
\hline Pos: $1: 7$ & Innsett & Dato: 21.06 .2008 & Avd: SURG & Kan redig & & Ikke fer & & & & & & \\
\hline
\end{tabular}

Figure 1. Screenshot of the nursing plan.

By clicking on the menu item "New diagnosis" in the action bar in the care plan, the system provided a new search window where the classifications were listed, and enabled the users to search and select a NANDA diagnosis in order to make it available in the care plan. The same procedure was used to create an intervention, where NIC classifications were available in the system. However, it was also possible to use free-text diagnosis instead of or in addition to NANDA and NIC.

During 2005, the electronic nursing module was put to use at the hospital, and the Department of Special Psychiatry was one of these pilot departments. During the implementation, a local project team was established that was in charge of preparing the nursing module, training end-users, and coordinating activities between the local and the central project at the hospital.

The implementation of the new EPR was a major change process because the ward moved from paper-based, unstructured documentation toward a highly structured electronic care plan with the use of international classifications. The paper-based documentation was widely characterized as incomplete, inaccurate, and subjective, and it used local jargon in the reports. In addition, they were largely dependent on oral communication for sharing information across the nursing team. Consequently, the staff was highly motivated to adopt the new computer-based system as a step toward improving the quality of nursing documentation. As a strategy for the implementation of the electronic nursing module, it was decided that using nursing terminologies was mandatory. The main motivation was to provide a language for describing both planned and performed care in their daily work practices. Key users at the ward assumed that mandatory use would give nurses and social workers a chance to become familiar with the classifications, as well as how they could be 
used in daily practice. Accordingly, mandatory use was intended to give the users a qualified choice and provide more specific language for describing patient problems and planned interventions. The use of the nursing module required new knowledge and skills, and education and guidance was a priority during the implementation period. Six super users at the ward were responsible for organizing and carrying out the training and supervision of all employees. The recruitment of super users was based on interest and expertise in the use of computer based systems and they received one day training in basic use of EPR and one day training in the use of care plan. Despite some sceptics and reluctance at the start, experiences during the first year showed that the care plans were used in daily practice and that classifications were used extensively (Table 1). Gradually, classifications became embedded in local practice and were used as a support in daily documentation, professional meetings, and discussions.

\subsection{Results from the frequency analysis (2008)}

After one year of use, it was evident that users still had some difficulty in finding appropriate classifications to describe all kinds of nursing diagnoses and interventions. Based on these observations, the management decided to loosen up its implementation strategy and give the users the opportunity to choose their own local terms to describe nursing diagnoses and interventions. As a result, after the change from mandatory to voluntary use of classifications, the use of NANDA decreased. However, at the same time, many of the local terms seemed to share some correspondence with NANDA terms.

To investigate the extent of this, we conducted an analysis based on reports from the EPR during the spring of 2008. We investigated a) the incidence of NANDA diagnoses and locally developed diagnoses, and (b) whether the local diagnosis could be mapped to a NANDA diagnosis. The study addresses the incidence of NANDA in the first period (April 2005-March 2006) and the second period (2007). The figures in the table indicate a clear decline in the use of NANDA diagnoses.

Table 1. Distribution of NANDA and locally developed diagnoses

\begin{tabular}{lll}
\hline & $2005 / 2006$ & 2007 \\
\hline Total number diagnoses & 805 & 909 \\
NANDA diagnoses & $756(93.9 \%)$ & $526(57.9 \%)$ \\
Locally-developed diagnoses & $49(6.1 \%)$ & $383(42.1 \%)$ \\
\hline
\end{tabular}

The next step was to determine to what extent the local diagnosis could be mapped to a NANDA diagnosis. Terms from the local diagnosis were compared with terms in NANDA 
and characterized as "Same," "Similar," "Broader," "Narrower," and "No Match" [54]. Validation of consistency and accuracy was done by two of the authors, both nurses, performing the same mapping separately. After completing the mapping, we compared our assessments and found that a minor degree of inconsistency occurred when a local diagnosis could be mapped to several NANDA diagnoses. After a discussion of the causes for this we reached almost $100 \%$ consensus [54]. We found that around $70 \%$ of all local diagnoses could be mapped to a NANDA diagnosis.

Table 2. Characterization of local diagnoses in 2007

\begin{tabular}{ll}
\hline Total local diagnoses & 383 \\
Same & $41(10.7 \%)$ \\
Similar & $80(20.9 \%)$ \\
Broader & $24(6.3 \%)$ \\
Narrower & $124(32.4 \%)$ \\
No match & $114(29.8 \%)$
\end{tabular}

In the "No Match" category (29.8\%), $9.9 \%$ could not be mapped to NANDA due to limitations in NANDA. Examples are nursing diagnoses related to emotions, aggression, and euphoria; adverse effects of medication; and problems related to allergies. Furthermore, we found a group of local codes $(8.4 \%)$ that could not be interpreted as a nursing diagnosis. This category, together with procedure, intervention, observation, and medical diagnoses, is regarded as reflecting errors and not nursing diagnoses.

Table 3. Distribution of NANDA and locally developed diagnoses mapped to NANDA

\begin{tabular}{lll}
\hline & \multicolumn{1}{c|}{$\mathbf{2 0 0 5} / \mathbf{2 0 0 6}$} & \multicolumn{1}{c}{$\mathbf{2 0 0 7}$} \\
\hline Total number of diagnoses & 805 & 909 \\
$\begin{array}{l}\text { NANDA diagnoses }+ \text { local } \\
\text { diagnoses mapped to NANDA }\end{array}$ & $791(98.3 \%)$ & $795(87.5 \%)$ \\
Locally developed diagnoses & $14(1.7 \%)$ & $114(12.5 \%)$ \\
\hline
\end{tabular}

If we combine the number of NANDA diagnoses with the number of local diagnoses that could be mapped to NANDA in 2007 , we get $87.5 \%$. If we subtract diagnoses that were considered errors, $8.4 \%$ (76 diagnoses), we get $95.5 \%$. This implies that NANDA 
diagnoses in use and local diagnoses mapped to NANDA constituted $95.5 \%$ of all nursing diagnoses documented in 2007, a strikingly high coverage.

\subsection{Results from the longitudinal case study (2005-2010)}

So, how do we explain the high degree of local terms that were similar to NANDA, particularly as many nursing professionals complain about the American bias and how remote the system is from local practice [33, 35]. Based on our longitudinal data, we wanted to dig deeper on this.

We found that NANDA and similar local terms had been tightly embedded in local practice and used for the support of daily documentation, professional meetings, and discussions. Already from the implementation of the nursing module at the psychogeriatric ward in 2005, there has been a continuous focus on the electronic care plan, where the nurse in charge of professional development regularly offered guidance and support to the individual user to ensure the quality of nursing documentation. Some users needed support to use the care plan and others needed professional assistance to find words to describe problems and interventions in the care plan. In any case, the care plan was always the starting point for this guidance.

"It is great to use the care plan as a basis for discussing the individual patient." (Nurse)

The nursing staff also discussed terms and learned from each other. When they sat together in the nursing office, they often discussed issues in the care plan, for instance, what the actual content of a NANDA term means. Often, the two nurses responsible for a particular patient work together with the patient's care plan.

"For sure, we have been more confident in using classifications, but still we discuss the meanings of diagnoses and give feedback to each other." (Nurse)

If they couldn't find the right concept in NANDA, they discussed how to formulate the problem and often come up with an alternative (but still related) concept. An example is "agitated behaviour," which is not included in NANDA. The closest term is "risk for violence," but the nurses felt that this would send a misleading signal, implying that the patient is intentionally violent. Instead, they used the term "risk for aggressive behaviour." This term is similar to NANDA's term, but the local one seems more appropriate in practical use. Nurses also learned from each other by reading existing care plans as illustrated in the quote below:

"I often look in the care plan of other patients who have the same problem to decide what terms to use." (Nurse) 
During handover meetings, the care plan was always displayed on the wall using a video projector. In addition, care plans and classifications had been the subject of clinical reflection and discussions.

After several years of training, nurses had become accustomed to using terms from NANDA. They used some terms frequently, and these became a part of the vocabulary at the ward. NANDA terms such as "bathing/hygiene self-care deficit" were often used and corresponded with the professional terminology used locally. However, users often experienced that a patient lacked self-care in relation to several function areas, or on a general basis that was not limited to bathing/hygiene or eating as defined in NANDA. As a result, they often chose a free-text diagnosis and write, "self-care deficit" or "self-care deficit in all function areas."

"I think it is okay to work with classification because it is easier to change something that already exists than to reinvent the wheel. If I find a classification that does not completely fit the situation of the patient, it is still easy to take the classification as a starting point. Instead of 'self-care deficit, eating,' I can write, 'self-care deficit, all function areas."'(Nurse)

Other examples of frequently used NANDA terminology were "risk for falls" and "impaired physical mobility." If used separately, these terms represent two different NANDA diagnoses. However, some nurses preferred to link several terms into one nursing diagnosis, such as "impaired physical mobility with risk for fall". In this case, they had become familiar with two different NANDA terms and combined them to form a local diagnosis. One observed problem with NANDA was that the terms are fragmentary. First, patients often had many different problems that were related to one other. In many cases, it was therefore appropriate to relate to symptoms and causes. Second, many patients had several problems, and the care plan therefore become very long and complex if the information was fragmented. Therefore, through experience and discussions, it became common and more effective to link different problems with one local nursing diagnosis.

Adding an explanatory comment to a NANDA diagnosis was also recommended. For instance, "anxiety" is a commonly used NANDA diagnosis, but it needed a more specific explanation, such as "anxiety related to depression." To document this in the electronic care plan, the users first had to search for the NANDA diagnosis, "anxiety," and then add, "Related to depression" in the comment field. Entering this information in the care plan required intensive mouse-clicking through various windows, dialogue boxes, and menus within the application. It required 10 mouse-clicks to add a NANDA diagnosis and 5 mouse-clicks to add a local diagnosis. After becoming more familiar with the terms of NANDA, some found it easier to simply add the whole entry as a local diagnosis. In this 
way, development of new concepts and expressions occurred. Gradually, several local terms evolved that were used frequently in the ward.

"One day I said to my colleague, 'We should write down all these local terms that are buzzing around.' So we sat down and created a local list of terms that we often use instead of NANDA." (Nurse)

To make these concepts available in the documentation process, the local terms were collected in a paper-based list. Later, some users had the idea of adding the list of concepts as a document in the nursing plan. The nursing module offered functionality for adding a "standard care plan" as a guideline. This functionality was used in the local practice to maintain the list of locally developed diagnoses. From the user interface in the care plan, it was possible to present the "Standard Care Plan" (i.e., the list of local diagnoses) on the screen. Then, it was an easy matter to choose those terms suitable for explaining the patient's condition. Many nurses found the local list a valuable support when they wanted to create a care plan or when they were looking for words or expressions to describe a patient's problems.

\section{Discussion}

\subsection{Establishing standardised terminologies in practice - a process of co-construction}

Several studies have pointed out how the development of standards is co-constructed with an emerging work practice [40]. Still, very few have taken a mixed method approach and illustrated the extent (frequency analysis) and content (the longitudinal case study) of the mutual shaping process empirically. The locally developed codes in this case were shaped by a mutual shaping process: the global NANDA codes, specific local needs as well as limitations and affordances of the care plan.

Previous studies have identified a semantic gap when using nursing classifications in clinical practice [see, for instance, 22, 25, 55]. These studies have focused mainly on similarities and differences between the standardised terminologies and concepts used in daily practice. In comparison, our study moves beyond this by identifying the degree of resemblance between the co-constructed local codes and NANDA as well how these emerged and how they were used.

We found that, in spite of the personnel's increased freedom to choose their own local codes, as many as $70 \%$ of the locally developed codes were more or less consistent with NANDA, implying that in total $95.5 \%$ of all nursing diagnoses were consistent with NANDA. This clearly indicates that the locally-developed codes were shaped by both local 
practice and NANDA. Here it is quite interesting that even if the users had the freedom to choose for themselves, they still lent on NANDA diagnoses or locally developed terms.

In addition, we are also able to illustrate how the use of co-constructed codes gradually emerged over time, which to a large degree has been missing in much of the medical informatics literature [13]. Overall, the users at the ward experienced improved documentation of nursing after implementation of the new system. The use of terminologies enabled a more consistent and shared language for describing nursing and for supporting coordination of the care process among the care team. However, increasingly the nursing diagnoses were not selected from the NANDA list in the system but rather adjusted and modified as locally developed concepts. Some patients often had several complex and interrelated problems, and therefore it was common to combine several NANDA diagnoses to represent one local concept. Another issue was nuances of the language that could have different meanings across different contexts. For example, some nurses at the ward thought that the NANDA diagnosis "risk for other-directed violence" was too strong an expression to use in relation to an older patient with agitated behaviour because it did not fit people who suffered from cognitive impairment and reduced understanding.

This illustrates the tension between semantic interoperability, which is supposed to guarantee an unambiguous exchange of meaning between humans [56] and the local diversity of nursing information. Consequently, interoperability is also a socio-technical challenge. For example, "wound" was a locally developed diagnosis that could be mapped to the NANDA diagnosis "impaired skin integrity." This is a rather broad diagnosis that covers all kinds of disruption or invasion of skin surface. Yet the term "wound" makes more sense in local practice, since it provides a more specific description of the problem and has been part of the local language for many years. The practice of elaborating the given categories through specialization illustrates the issue about the level of granularity. On the one hand, it is a striving for a "one size fits all" solution that covers all aspects of nursing. On the other hand, a danger is that the categories are too broad and do not fit anything accurately.

\subsection{Moving out of the local context}

While our study has encompassed the extent, formation and use of standardized terminologies, we have focused on one particular context - a psychogeriatric ward. This has enabled us to focus on transformation in this context over time. However we don't claim a general validity of our mapping exercise (i.e. $95.5 \%$ similarity) when including 
different contexts. Still we believe that our argument on how global terminologies and practice are co-constructed has validity beyond the psychogeriatric ward.

Our study points out that global terminologies are inevitably bound to local practice. Accordingly, when we know that nursing practice in general is comprehensive and diverse, the high expectations of comparing diagnoses across different wards may not come through. $[25,57]$. The use of NANDA may have different meaning in various contexts. For example, the NANDA diagnosis "anxiety" typically has a different meaning used in a psychiatric department than in a surgical department. In the psychogeriatric ward, anxiety is a common nursing diagnosis, since many of the patients are suffering from depression or dementia. In comparison, when the nursing diagnosis "anxiety" is used in a different context, such as to describe patients who are anxious before surgery, it is a completely different problem. This illustrates the semantic gap that may occur within a specific domain as well as across diverse practices. Consequently, sharing information and knowledge in nursing is not just a matter of transferring, but also a process of translating and transforming domain-specific knowledge [41]. Hence we believe that our finding on coconstruction in a longitudinal perspective between global terminologies and local practice also may play out between two different contexts (i.e. surgery and psychiatry) where the global NANDA terminologies is shaping and shaped by the different practices involved.

So where does this take us regarding sharing of codes across contexts? Basically, we argue that the observed tinkering with terminology standards is not a failing, but a prerequisite for it to function effectively in the first place [43]. This also illustrates a key point: the translation process between contexts/wards is associated with some costs and efforts. Generally, we believe that some translation processes could be done on a routine basis, where the benefits and costs are compared and weighed. However, we also believe that in some instances, the use of codes in different practices must be carefully examined on an individual basis before being translated because of a very different use (e.g., anxiety) [58]. The good thing about it is that the need to do this is not immediately present. In daily practice, this is not a problem since clinical practice basically is bound to a specific site and situation, and nurses have the ability to translate or transform classifications, such as "anxiety," across domain-specific boundaries.

\subsection{The collective initiative}

Our longitudinal approach has illustrated how the co-constructed concepts increasingly have emerged through a collective process. You may consider how the users searched the NANDA list, and then modified the NANDA codes according to the actual situation. Another example is how nurses' changed NANDA diagnoses slightly to establish a link 
between diagnosis and intervention in the care plan as a way of generating coherence in the care plan. There was a gradual development in which increased knowledge of NANDA resulted in increased awareness of using the language to describe nursing, which in turn led to increased development of local nursing diagnoses.

As a part of this collective effort, we draw attention to a surprising similarity with folksonomies, i.e., how social tagging and social classification can empower a relatively large group of participants. The dynamic and structure of folksonomies are characterized by frequency of tagging, the information value of tags, and a feedback cycle between the elements involved in the process (users, tags, resources) [59]. Such a system is more or less self-evolving, self-maintaining, and flexible. As with folksonomies, the local concepts in the psychogeriatric ward have emerged through a bottom-up process based on collegial collaboration. This collaboration has been grounded in face-to-face interaction among the staff in the process of formalizing the electronic nursing documentation.

A key issue of this co-constructive perspective with the use of local classifications (i.e., folksonomies) is how it enabled information sharing and development of local knowledge. For instance, in this case the nursing care plan including the terminologies had a key role in professional meetings (morning meetings among the nurse team), where it was displayed on a wall screen and enabled co-operative information sharing. Accordingly, the classification practices were embedded in the social structures in the ward, where the daily use of nursing terminologies became foundation for a learning process.

\section{Conclusion}

In this study, we have demonstrated how the use of standardized terminologies enable a more complete and accurate representation of nursing information in the EPR and thus facilitate quality and safety in patient care. However, adoption and use of standardized terminologies is not just a "plug and play" solution. From a purely technical perspective, a unified language is important for clear and non-redundant representation of concepts. However, health care is typically characterized by multiplicity and diversity. Semantic interoperability is important, but as we have argued, not sufficient to integrate standardized terminologies in nursing practice. We have used a pragmatic approach and a co-constructed perspective to study the interrelationships between global standards and local practices. First, the study shows a surprisingly high correlation between NANDA and local classifications. Second, the study shows how local classifications evolved during long-term use and how local classifications emerged as a supplement to formal classifications. An 
interesting point was the mutual dependence between NANDA and local classifications and how this was an iteration that evolved through long-term use. Third, we have emphasized the multiplicity of nursing practice and how this co-construction was used to bridge the gap between global classification and local needs. Finally, we suggest that this "artful standardization" can be used as an advantage and opportunity in which local knowledge and agency is used as a means to individualize the content of the standard.

\section{References}

[1] Kohn LT, Corrigan J, Donaldson MS. To err is human: building a safer health system. Washington, D.C.: National Academy Press; 2000.

[2] Bates DW, Gawande AA. Improving safety with information technology. New England Journal of Medicine2003;348(25):2526-34.

[3] Poon EG, Keohane CA, Yoon CS, Ditmore M, Bane A, Levtzion-Korach O, Moniz T, Rothschild JM, Kachalia AB, Hayes J. Effect of bar-code technology on the safety of medication administration. The New England Journal of Medicine2010;362(18):1698 - 707.

[4] Ash JS, Berg M, Coiera E. Some unintended consequences of information technology in health care: the nature of patient care information system-related errors. Journal of the American Medical Informatics Association2004;11(2):104-12.

[5] Koppel R, Wetterneck T, Telles JL, Karsh BT. Workarounds to barcode medication administration systems: their occurrences, causes, and threats to patient safety. Journal of the American Medical Informatics Association2008;15(4):408-23.

[6] Campbell EM, Sittig DF, Ash JS, Guappone KP, Dykstra RH. Types of unintended consequences related to computerized provider order entry. Journal of the American Medical Informatics Association2006;13(5):547.

[7] Aarts J, Ash J, Berg M. Extending the understanding of computerized physician order entry: implications for professional collaboration, workflow and quality of care. International Journal of Medical Informatics2007;76:S4-S13.

[8] Bakken S, Cimino JJ, Hripcsak G. Promoting patient safety and enabling evidence-based practice through informatics. Medical care2004;42(2):II.

[9] Aspden P. Patient safety: achieving a new standard for care: Natl Academy Pr; 2004.

[10]Häyrinen, K., Saranto K, Nykänen P. Definition, structure, content, use and impacts of electronic health records: a review of the research literature. International Journal of Medical Informatics2008;77(5):291-304.

[11]Berg M, Timmermans S. Order and their others: On the constitution of universalities in medical work. Configurations2000;8(1):3162.

[12] Weaver CA, Warren JJ, Delaney C. Bedside, classroom and bench: Collaborative strategies to generate evidence-based knowledge for nursing practice. International Journal of Medical Informatics2005;74(11-12):989-99.

[13] Häyrinen K, Lammintakanen J, Saranto K. Evaluation of electronic nursing documentation--Nursing process model and standardized terminologies as keys to visible and transparent nursing. International Journal of Medical Informatics2010;79(8):554-64.

[14] ISO. Health informatics - Electronic health record - Definition, scope, and context. ISO TC 215. 2005.

[15] Cubas MR, Denipote AG, Malucelli A, da Nobrega MM. The ISO 18.104: 2003 as integrative model of nursing terminologies. Revista Latino-Americana de Enfermagem2010 Jul-Aug;18(4):669-74.

[16] Kalra D. Electronic health record standards. IMIA Yearbook of Medical Informatics2006;2006:136-44.

[17] Lenz R, Beyer M, Kuhn KA. Semantic integration in healthcare networks. International Journal of Medical Informatics2007;76(23):201-7.

[18] Knaup P, Bott O, Kohl C, Lovis C, Garde S. Electronic patient records: moving from islands and bridges towards electronic health records for continuity of care. Yearb Med Inform2007:34-46.

[19] Thoroddsen A, Saranto K, Ehrenberg A, Sermeus W. Models, standards and structures of nursing documentation in European countries. Studies in health technology and informatics2009;146:327.

[20] Hardiker NR, Hoy D, Casey A. Standards for nursing terminology. Journal of the American Medical Informatics Association2000;7(6):523-8.

[21] Müller-Staub M, Lavin MA, Needham I, van Achterberg T. Meeting the criteria of a nursing diagnosis classification: Evaluation of ICNP $\neg Æ$, ICF, NANDA and ZEFP. International journal of nursing studies2007;44(5):702-13.

[22] Goossen W. Cross- Mapping Between Three Terminologies With the International Standard Nursing Reference Terminology Model. International Journal of Nursing Terminologies and Classifications2006;17(4):153-64. 
[23] Coenen A, Kim TY. Development of terminology subsets using ICNP®. International Journal of Medical Informatics2010;79(7):530-8.

[24] Hardiker NR, Casey A, Coenen A, Konicek D, editors. Mutual enhancement of diverse terminologies. AMIA Annual Symposium; 2006; Washington DC, USA: American Medical Informatics Association.

[25] Kim TY, Coenen A, Hardiker N. Semantic mappings and locality of nursing diagnostic concepts in UMLS. Journal of biomedical informatics2011.

[26] Timmermans S, Berg M. The gold standard: The challenge of evidence-based medicine and standardization in health care. Philadelphia: Temple Univ Pr; 2003.

[27] Gordon M. Nursing nomenclature and classification system development. Online Journal of Issues in Nursing1998;30.

[28] Saba V, Hovenga E, Coenen A, McCormick KA, Bakken S. Nursing Language - Terminology models for nurses. ISO Bulletin September 20032003.

[29] International N. Nursing Diagnosis: Definition \& Classification. Philadelphia: NANDA International; 2007.

[30] Hanseth O, Monteiro E, Hatling M. Developing information infrastructure: The tension between standardization and flexibility. Science, Technology \& Human Values 1996;21(4):407.

[31] Schmidt K, Bannon L. Taking CSCW seriously. Computer Supported Cooperative Work (CSCW)1992;1(1):7-40

[32] Bakken S, Hyun S, Friedman C, Johnson S. ISO reference terminology models for nursing: applicability for natural language processing of nursing narratives. International Journal of Medical Informatics2005;74(7-8):615-22.

[33] Benner P. Designing formal classification systems to better articulate knowledge, skills, and meanings in nursing practice. Am J Crit Care2004 Sep;13(5):426-30.

[34] Bowker G, Star S. Sorting things out: classification and its consequences. Cambrigde: The MIT Press; 2000.

[35] Clark J. The international classification for nursing practice project. Online Journal of Issues in Nursing 1998;30.

[36] Moen A, Henry SB, Warren JJ. Representing nursing judgements in the electronic health record. J Adv Nurs1999 Oct;30(4):990-7.

[37] Dykes PC, Kim H, Goldsmith DM, Choi J, Esumi K, Goldberg HS. The adequacy of ICNP version 1.0 as a representational model for electronic nursing assessment documentation. Journal of the American Medical Informatics Association2009;16(2):238-46.

[38] Kim TY, Coenen A. Toward harmonising WHO International Classifications: a nursing perspective. Informatics for health \& social care2011;36(1):35.

[39] Hardiker NR, Rector AL. Structural validation of nursing terminologies. Journal of the American Medical Informatics Association2001;8(3):212-21.

[40]Ellingsen G, Monteiro E, Munkvold G. Standardisation of work: co-constructed practice. The Information Society2007;23(5):309 26.

[41]Carlile PR. Transferring, translating, and transforming: An integrative framework for managing knowledge across boundaries. Organization Science2004;15(5):555-68

[42] Goodwin C. Professional vision. American anthropologist1994;96(3):606-33.

[43] Timmermans S, Berg M. Standardization in action: Achieving local universality through medical protocols. Social studies of science 1997;27(2):273-305.

[44]Berg M, Goorman E. The contextual nature of medical information. International Journal of Medical Informatics1999;56(1-3):51-60.

[45] Orlikowski WJ. Categories: concept, content, and context. Computer Supported Cooperative Work (CSCW)1994;3(1):73-8.

[46] Ure J, Procter R, Lin Y, Hartswood M, Anderson S, Lloyd S, Wardlaw J, Gonzalez-Velez H, Ho K. The Development of Data Infrastructures for eHealth: A Socio-Technical Perspective. Journal of the Association for Information Systems2009;10(5):415 - 29.

[47]Hepsø V, Monteiro E, Rolland K. Ecologies of e-Infrastructures. Journal of the AIS2009;10(5):430-46.

[48] Peters I. Folksonomies: Indexing and Retrieval in the Web 2.0. Berlin: KG Saur Verlag Gmbh \& Co; 2009.

[49] Walsham G. Interpretive case studies in IS research: nature and method. Eur JInf Systs 1995;4:74 - 81.

[50] Orlikowski W, Baroudi J. Studying information technology in organizations: Research approaches and assumptions. Information systems research1991;2(1):1-28.

[51] Klein H, Myers MD. A set of principles for conducting and evaluating interpretive field studies in information system. MIS Quarterly 1999;23(1):67 - 94.

[52]Forsythe DE. ilt's Just a Matter of Common Senseî: Ethnography as Invisible Work. Computer Supported Cooperative Work (CSCW)1999;8(1):127-45.

[53] Yin RK. Case study research: Design and methods: Sage publications, INC; 2009.

[54]Zielstorff RD, Tronni C, Basque J, Griffin LR, Welebob EM. Mapping nursing diagnosis nomenclatures for coordinated care. Image J Nurs Sch1998;30(4):369-73.

[55]Ehnfors M, Florin J, Ehrenberg A. Applicability of the International Classification of Nursing Practice (ICNP) in the Areas of Nutrition and Skin Care. International Journal of Nursing Terminologies and Classifications2003;14(1):5-18.

[56] Mead CN. Data Interchange Standards in Healthcare IT-Computable Semantic Interoperability: Now Possible but Still Difficult. Do We Really Need a Better Mousetrap? Journal of Healthcare Information Management2006;20(1):71. 
[57] Hovenga E, Garde S, Heard S. Nursing constraint models for electronic health records: a vision for domain knowledge governance. International Journal of Medical Informatics2005;74(11-12):886-98.

[58] Mol A. The body multiple: ontology in medical practice: Duke University Press; 2002.

[59] Halpin H, Robu V, Shepherd H, editors. The complex dynamics of collaborative tagging. WWW2007; 2007; Canada: ACM. 